\title{
THE OKU CONCEPT AND THE SEQUENTIAL DEPTH
}

\author{
Author(s) / Auteur(s) : \\ Jean-Luc CAPRON \\ Faculté d'architecture, d'ingénierie architecturale, d'urbanisme (LOCI) \\ Université catholique de Louvain (UCL) \\ jean-luc.capron@uclouvain.be
}

Keywords / Mots-clés :

architecture, oku, japanese, space, perception

In the field of architecture, the Japanese spatial concept $o k u$, may be approximated by the Westerners as a "depth strategy". This meets the ma concept which may be approximated as a "negative space." If these two concepts refer to time, their relationship to the dynamics of perception is characterized by a static subject for the concept of $m a$, and by a motion subject for the concept of $o k u$.

Architecture acquires its reality when perceived and used by human beings. Therefore, issues of a preliminary research on the perceptions and uses of space are the knowledge of the modalities of the perceptions and uses that shape mental spatial constructions, which in turn make our relation to the built environment. Acquiring such a knowledge assumes that we agree to focus - at least at a first step - on the analysis of spatio-temporal instantaneous rooted in the hic et nunc dimension, implicit to any action.

This approach requires to develop specific tools to address the issue of the relationship that links human beings to the built environment. Here, the concept of system is a conceptual tool, which aims to highlight the interactions between the elements of a complex whole. In such a context, a systemic approach is a tool that offers many opportunities.

Since the relationships between the components of a system are not unidirectional but bidirectional, a representation based on communication theory may be used to represent information in a formoriented

vector. This vector is characterized by its points of origin and destination, as well as the amount of information transmitted or received. As some information lead to actions and re-actions, time sequencing factors would be added to the main features of the analysed relationships. Thus, these actions can also be considered as vectors characterized in a manner similar to information, but without being their reciprocal.

As the degree of organization in a system depends on the qualitative and quantitative information available. And as the human being perceives the built environment by means of sensory receptors and sociocultural filters, ... one can state that the built environment system is thus perceived by an external system. Therefore, the amount of perceived organization depends on the occurrences between the "observer" and the "observed". The information exchange process from one system to another can be considered as the transfer of an organization. And the nature of the information may be considered as a pattern.

The interface between humans and the built environment is carried out by systems of interactions between the activity of the human being and the constraints of the built environment. The systems 
discussed here are open and non-homogeneous. Open in that they are permeable to the environment external to the system and not homogeneous in that the elements which are interrelated are of different natures.

To do this, the concept of micro-system has been developed to make possible the integration of interfaces, with a significant physio-psychological impact on user behaviour. The definition of a microsystem

requires isolating components, endogenous and exogenous, as well as the subsystems and their relationships. In architecture, the identification of a micro-system can be made on the basis of factors such as the built environment stimuli, or perceptual and cognitive modalities via which the human being builds a mental picture that helps to interact with the surroundings and induces behaviour.

Such methodological tools help to characterize the "sequential depth" associated with the concept of $o k u$ which lead the journey to the "inner space". A spatial concatenation in which one enters, actually or

virtually, according to a ritual journey through the spatial structure that binds the various places in the respect of the specificity of use. Performing the two spatio-temporal mechanisms - not only by extending space, but also by generating a sense of depth -, suggesting a relationship with an inaccessible space that refers to "the impression of distance". 